Bull. Austral. Math. Soc.

$60 \mathrm{H} 40,46 \mathrm{~F} 25,60 \mathrm{H} 30,60 \mathrm{G} 20,47 \mathrm{D} 06,47 \mathrm{D} 62$

VOL. 68 (2003) [491-500]

\title{
DIFFERENTIAL EQUATIONS IN SPACES OF HILBERT SPACE VALUED DISTRIBUTIONS
}

\section{MaXim ALSHANSKY}

\begin{abstract}
A Gaussian measure is introduced on the space of Hilbert space valued tempered distributions. It is used to define a Hilbert space valued $Q$-Wiener process and a white noise process with a nuclear covariance operator $Q$. The proposed construction is used for solving operator-differential equations with additive noise with the operator coefficient generating an $n$-times integrated exponentially bounded semigroup.
\end{abstract}

\section{INTRODUCTION}

Let $X$ and $Y$ be separable Hilbert spaces. We denote by $D^{\prime}(X)$ the space of $X$ valued distributions defined on $D$, the space of infinitely differentiable functions with compact supports. By $D_{+}^{\prime}(X)$ we denote the subspace of distributions from $D^{\prime}(X)$ with supports bounded from below.

Any linear time-invariant dynamic system is fully determined by its state equation which can be written in the form

$$
P * U=F,
$$

where $P \in D_{+}^{\prime}(\mathcal{L}(X ; Y)), U \in D_{+}^{\prime}(X), F \in D_{+}^{\prime}(Y)$ (see [1]). The system is said to be invertible if there exists $G \in D_{+}^{\prime}(\mathcal{L}(Y ; X))$, the convolution inverse for $P$, so that the equalities $P * G=\delta \otimes I_{Y}$ and $G * P=\delta \otimes I_{X}$ hold. In this case formula $U=G * F$ yields the unique solution of (1) (see details in [1]).

One can model stochastic influence of the environment on the system by introducing an appropriately defined 'noise' term $W$ into the right-hand side of (1).

$$
P * U=F+W .
$$

A solution of the perturbed equation formally can be written in the form $U=Q *(F+W)$.

In this note we construct a Gaussian measure on the space of $H$-valued tempered distributions, where $H$ is a separable Hilbert space, using the approach of [3]. We use the approach of [2] to define $Q$-Wiener process and $Q$-white noise process as generalised processes with values in $H$ (where $Q: H \rightarrow H$ is a nuclear operator). This makes convolution $Q * B W$ well-defined for any linear bounded operator $B: H \rightarrow Y$ in the same sense as it is defined for Hilbert space valued distributions.

Received 11th June, 2003

Copyright Clearance Centre, Inc. Serial-fee code: 0004-9727/03 \$A2.00+0.00. 


\section{Preliminaries}

Consider a Gelfand triple

$$
S \subseteq S_{0} \subseteq S^{\prime}
$$

where $S_{0}=L^{2}(\mathbb{R}), S$ is the Schwartz space of rapidly decreasing test functions and $S^{\prime}$ is the space of corresponding tempered distributions.

Denote by $(\cdot, \cdot)_{0}$ and $\mid \cdot 10$ the inner product and the corresponding norm in $S_{0}$. Consider the linear operator $A:=-\left(d^{2} / d x^{2}\right)+x^{2}+1$. For all $p \in \mathbb{Z}, \xi \in S$ let $|\xi|_{p}=\left|A^{p} \xi\right|_{0}$. Let $(\cdot,)_{p}$ be the corresponding inner product and $S_{p}$ be the completion of $S$ with respect to $|\cdot|_{p}$. The space $S_{-p}$ is the dual of $S_{p}$ for each $p>0$. Then we have the following inclusions:

$$
S=\bigcap_{p \in \mathbb{N}} S_{p} \subset \cdots \subset S_{p+1} \subset S_{p} \subset \cdots \subset S_{0} \subset \ldots S_{-p} \subset S_{-p-1} \subset \cdots \subset \bigcup_{p \in \mathbb{N}} S_{p}=S^{\prime} .
$$

We denote by $\langle\omega, \xi\rangle$ the dual pairing of $\omega \in S^{\prime}$ and $\xi \in S$. For $\omega \in S_{0}$, we have $\langle\omega, \xi\rangle=(\omega, \xi)_{0}$. The space $S$ is a countably Hilbert nuclear space endowed with the projective limit topology. Its dual $S^{\prime}$ is the inductive limit of $\left\{S_{-p}, p \geqslant 1\right\}$.

Consider Hermite polynomials

$$
H_{n}(x)=(-1)^{n} e^{x^{2}} \frac{d^{n}}{d x^{n}} e^{-x^{2}}, \quad n=0,1,2, \ldots
$$

and the corresponding Hermite functions

$$
\xi_{n}(x)=\frac{1}{\pi^{1 / 4}(n !)^{1 / 2} 2^{n / 2}} H_{n}(x) e^{-\left(x^{2} / 2\right)}, \quad n=0,1,2, \ldots .
$$

The set $\left\{\xi_{n}\right\}_{n=0}^{\infty}$ is an orthonormal basis for $S_{0}$ and we have

$$
A \xi_{n}=(2 n+2) \xi_{n}, \quad n=0,1,2, \ldots
$$

For any $\xi \in S_{p}, p \in \mathbb{Z}$ we have

$$
|\xi|_{p}=\left(\sum_{n=0}^{\infty}(2 n+2)^{2 p}\left(\xi, \xi_{n}\right)_{0}^{2}\right)^{1 / 2} .
$$

Let $H$ be a separable Hilbert space with scalar product $(\cdot, \cdot)_{H}$ and the corresponding norm $\|\cdot\|_{H}$. Let $\left\{e_{n}\right\}_{n=1}^{\infty}$ be an orthonormal basis in $H$.

Consider tensor products of Hilbert spaces $S_{p} \otimes H$ for $p \in \mathbb{Z}$. Denote by $[\cdot, \cdot]_{p}$ the inner product in $S_{p} \otimes H$ and by $\|\cdot\|_{p}$ the corresponding norm. Since $\left\{\xi_{i} \otimes e_{j}\right\}_{i=0, j=1}^{\infty}$ is an orthonormal basis in $S_{0} \otimes H$, any $\eta \in S_{p} \otimes H$ admits the following unique representation

$$
\eta=\sum_{i=0 ; j=1}^{\infty} \eta_{i j}\left(\xi_{i} \otimes e_{j}\right)=\sum_{j=1}^{\infty} \eta_{j} \otimes e_{j}=\sum_{i=0}^{\infty} \xi_{i} \otimes h_{i},
$$


where $\eta_{i j}=\left[\eta, \xi_{i} \otimes e_{j}\right]_{0}, \eta_{j}=\sum_{i=0}^{\infty} \eta_{i j} \xi_{i} \in S_{p}, h_{i}=\sum_{j=1}^{\infty} \eta_{i j} e_{j} \in H$

We have

$$
\|\eta\|_{p}^{2}=\sum_{i=0 ; j=1}^{\infty} \eta_{i j}^{2}(2 i+2)^{2 p}=\sum_{j=1}^{\infty}\left|\eta_{j}\right|_{p}^{2}=\sum_{i=0}^{\infty}(2 i+2)^{2 p}\left\|h_{i}\right\|_{H}^{2}
$$

For the inner product in $S_{p} \otimes H$ we have

$$
[\eta, \theta]_{p}=\sum_{i=0 ; j=1}^{\infty} \eta_{i j} \theta_{i j}(2 i+2)^{2 p}=\sum_{j=1}^{\infty}\left(\eta_{j}, \theta_{j}\right)_{p}^{2}=\sum_{i=0}^{\infty}(2 i+2)^{2 p}\left(h_{i}, g_{i}\right)_{H}
$$

Consider tensor products $S \otimes H$ and $S^{\prime} \otimes H$. We have

$$
\begin{aligned}
S \otimes H=\bigcap_{p \in \mathbb{N}} S_{p} \otimes H \subset & \subset \subset S_{p+1} \otimes H \subset S_{p} \otimes H \subset \cdots \subset S_{0} \otimes H \subset \\
& \subset \ldots S_{-p} \otimes H \subset S_{-p-1} \otimes H \subset \cdots \subset \bigcup_{p \in \mathbb{N}} S_{p} \otimes H=S^{\prime} \otimes H .
\end{aligned}
$$

Clearly, $S \otimes H$ is a countably Hilbert space endowed with the projective limit topology, $S^{\prime} \otimes H$ is its dual and is the inductive limit of $\left\{S_{-p} \otimes H, p \geqslant 1\right\}$. Note that $S \otimes H$ is not a nuclear space.

Denote by $[\cdot, \cdot]$ the dual pairing of elements from $S^{\prime} \otimes H$ and $S \otimes H$. For any $\omega \in S^{\prime} \otimes H$ and $\eta \in S \otimes H$ with

$$
\omega=\sum_{i=0 ; j=1}^{\infty} \omega_{i j}\left(\xi_{i} \otimes e_{j}\right)=\sum_{j=1}^{\infty} \omega_{j} \otimes e_{j}=\sum_{i=0}^{\infty} \xi_{i} \otimes g_{i}, \omega_{i j} \in \mathbb{R}, \omega_{j} \in S^{\prime}, g_{i} \in H
$$

and

$$
\eta=\sum_{i=0 ; j=1}^{\infty} \eta_{i j}\left(\xi_{i} \otimes e_{j}\right)=\sum_{j=1}^{\infty} \eta_{j} \otimes e_{j}=\sum_{i=0}^{\infty} \xi_{i} \otimes h_{i}, \eta_{i j} \in \mathbb{R}, \eta_{j} \in S, h_{i} \in H
$$

we have

$$
[\omega, \eta]=\sum_{i=0, j=1}^{\infty} \omega_{i j} \eta_{i j}=\sum_{j=1}^{\infty}\left\langle\omega_{j}, \eta_{j}\right\rangle=\sum_{i=0}^{\infty}\left(g_{i}, h_{i}\right)_{H}
$$

In particular, if $\omega \in S_{0} \otimes H$, then $[\omega, \eta]=[\omega, \eta]_{0}$.

Now we numerate the elements of $\left\{\xi_{i} \otimes e_{j}\right\}_{i=0, j=1}^{\infty}$. Define $\varepsilon_{k}=\xi_{i} \otimes e_{j}$, where

$$
k=k(i, j)=1+2+\cdots+(i+j-1)+j=\frac{(i+j)^{2}+j-i}{2} .
$$

In this case we have

$$
j=j(k)=k-\frac{\mathcal{N}(k)(\mathcal{N}(k)-1)}{2}
$$


and

where

$$
i=i(k)=\frac{\mathcal{N}(k)(\mathcal{N}(k)+1)}{2}-k,
$$

$$
\mathcal{N}(k)=\max \left\{n \in \mathbb{N} \mid \sum_{i=1}^{n-1} i=\frac{n(n-1)}{2} \leqslant k\right\} .
$$

\section{3. $Q$-WHite NOISE MEASURE ON $S^{\prime} \otimes H$}

Let $Q$ be a linear operator in $H$, defined by

$$
Q x=\sum_{j=1}^{\infty} \sigma_{j}^{2}\left(x, e_{j}\right)_{H} e_{j}, \quad x \in H
$$

with $\sum_{j=1}^{\infty} \sigma_{j}^{2}<\infty$. It is positive, self-adjoint and nuclear.

Consider a functional on $S \otimes H$ defined by

$$
C_{Q}(\eta)=\exp \left\{-\frac{1}{2}[(I \otimes Q) \eta, \eta]_{0}\right\}, \quad \eta \in S \otimes H .
$$

Denote by $\mathfrak{B}$ the Borel $\sigma$-field in $S^{\prime} \otimes H$.

THEOREM 1. There exists a probability measure $\mathfrak{m}_{Q}$ on $\left(S^{\prime} \otimes H, \mathfrak{B}\right)$ such that

$$
C_{Q}(\eta)=\int_{S^{\prime} \otimes H} \exp \{i[\omega, \eta]\} d \mathfrak{m}_{Q}(\omega), \quad \eta \in S \otimes H
$$

Proof: Denote by $P_{\varepsilon_{1}, \ldots, \varepsilon_{n}}$ the projector from $S^{\prime} \otimes H$ onto $S p\left\{\varepsilon_{1}, \ldots, \varepsilon_{n}\right\}$ :

$$
P_{\varepsilon_{1}, \ldots, \varepsilon_{n}}: \omega=\sum_{k=1}^{\infty} \omega_{i(k), j(k)} \varepsilon_{k} \mapsto \sum_{k=1}^{n} \omega_{i(k), j(k)} \varepsilon_{k} .
$$

Let $\rho_{\varepsilon_{1}, \ldots, \varepsilon_{n}}: P_{\varepsilon_{1}, \ldots, \varepsilon_{n}}\left(S^{\prime} \otimes H\right) \rightarrow \mathbb{R}^{n}$ be the natural isomorphism. Denote by $\mathfrak{B}_{\varepsilon_{1}, \ldots, \varepsilon_{n}}$ the collection of subsets in $S^{\prime} \otimes H$ defined by $\mathfrak{B}_{\varepsilon_{1}, \ldots, \varepsilon_{n}}=P_{\varepsilon_{1} ; \ldots, \varepsilon_{n}}^{-1} \rho_{\varepsilon_{1}, \ldots, \varepsilon_{n}}^{-1}\left(\mathcal{B}\left(\mathbb{R}^{n}\right)\right)$, where $\mathcal{B}\left(\mathbb{R}^{n}\right)$ is the Borel $\sigma$-field in $\mathbb{R}^{n}$. It consists of all sets of the form

$$
A=\left\{\omega=\sum_{k=1}^{\infty} \omega_{i(k), j(k)} \varepsilon_{k} \in S^{\prime} \otimes H \mid\left(\omega_{i(1), j(1)}, \ldots, \omega_{i(n), j(n)}\right) \in B\right\}, B \in \mathcal{B}\left(\mathbb{R}^{n}\right) .
$$

Define

$$
C_{\varepsilon_{1}, \ldots, \varepsilon_{n}}(\bar{z})=C_{Q}\left(z_{1} \varepsilon_{1}+\cdots+z_{n} \varepsilon_{n}\right), \quad \bar{z}=\left(z_{1}, \ldots, z_{n}\right) \in \mathbb{R}^{n} .
$$

For any $n \in \mathbb{N}, C_{\varepsilon_{1}, \ldots, \varepsilon_{n}}$ is a continuous positive-definite functional on $\mathbb{R}^{n}$ with $C_{\varepsilon_{1}, \ldots, \varepsilon_{n}}(0)$ $=1$. Therefore by Bochner's theorem it is a characteristic functional of a probability measure $m_{\varepsilon_{1}, \ldots, \varepsilon_{n}}$ on the measurable space $\left(\mathbb{R}^{n}, \mathcal{B}\left(\mathbb{R}^{n}\right)\right)$, so that

$$
C_{\varepsilon_{1}, \ldots, \varepsilon_{n}}(\bar{z})=\int_{\mathbb{R}^{n}} \exp \{i(\bar{x}, \bar{z})\} d m_{\varepsilon_{1}, \ldots, \varepsilon_{n}}(\bar{x}), \quad \bar{z} \in \mathbb{R}^{n} .
$$


Let $\mathfrak{m}_{\varepsilon_{1}, \ldots, \varepsilon_{n}}$ be a probability measure on $\left(S^{\prime} \otimes H, \mathfrak{B}_{\varepsilon_{1}, \ldots, \varepsilon_{n}}\right)$ defined by

$$
\mathfrak{m}_{\varepsilon_{1}, \ldots, \varepsilon_{n}}(A)=m_{\varepsilon_{1}, \ldots, \varepsilon_{n}}(B), A \in \mathfrak{B}_{\varepsilon_{1}, \ldots, \varepsilon_{n}}, A=P_{\varepsilon_{1}, \ldots, \varepsilon_{n}}^{-1} \rho_{\varepsilon_{1}, \ldots, \varepsilon_{n}}^{-1}(B), \quad B \in \mathcal{B}\left(\mathbb{R}^{n}\right) .
$$

It is not difficult to see that $\left\{m_{\varepsilon_{1}, \ldots, \varepsilon_{n}}\right\}_{n=1}^{\infty}$ is a consistent family of measures. Therefore by Kolmogorov's theorem there exists a probability space $(\Omega, \mathcal{F}, P)$ and a sequence of random variables $\left\{X_{n}\right\}_{n=1}^{\infty}$ such that

$$
m_{\varepsilon_{1}, \ldots, \varepsilon_{n}}=P\left(\bar{X}_{n}^{-1}\right) \text { with } \bar{X}_{n}=\left(X_{1}, \ldots, X_{n}\right), n=1,2, \ldots,
$$

and we have

$$
\begin{aligned}
C_{\varepsilon_{1}, \ldots, \varepsilon_{n}}(\bar{z}) & =\int_{\mathbb{R}^{n}} \exp \{i(\bar{x}, \bar{z})\} d m_{\varepsilon_{1}, \ldots, \varepsilon_{n}}(\bar{x}) \\
& =\int_{S^{\prime} \otimes H} \exp \left\{i\left[\omega, z_{1} \varepsilon_{1}+\cdots+z_{n} \varepsilon_{n}\right]\right\} d \mathfrak{m}_{\varepsilon_{1}, \ldots, \varepsilon_{n}}(\omega) \\
& =\int_{\Omega} \exp i\left(\bar{X}_{n}, \bar{z}\right) d P .
\end{aligned}
$$

LEMma 1. For any $\varepsilon>0$ there exists $k_{0} \in \mathbb{N}$ such that for any $p \in \mathbb{N}$

$$
\int_{\Omega} \exp \left\{-\frac{1}{2} \sum_{k=k_{0}}^{\infty}(2 i(k)+2)^{-2 p} X_{k}^{2}\right\} d P>1-\varepsilon .
$$

ProOF: For any $m, l \in \mathbb{N}$ with $m<l$ we have

$$
\begin{aligned}
& \int_{\Omega} \exp \left\{-\frac{1}{2} \sum_{k=m}^{l}(2 i(k)+2)^{-2 p} X_{k}^{2}\right\} d P \\
& =\int_{\Omega} \int_{\mathbb{R}^{l-m}} \exp \left\{i \sum_{k=m}^{l} X_{k} z_{k}\right\} \frac{\prod_{k=m}^{l}(2 i(k)+2)^{p}}{(2 \pi)^{(l-m) / 2)}} \exp \left\{-\frac{1}{2} \sum_{k=m}^{l}(2 i(k)+2)^{2 p} z_{k}^{2}\right\} d \bar{z} d P \\
& =\frac{\prod_{k=m}^{l}(2 i(k)+2)^{p}}{(2 \pi)^{(l-m) / 2)}} \int_{\mathbb{R}^{l-m}} C_{\varepsilon_{m}, \ldots, \varepsilon_{l}}\left(z_{m}, \ldots, z_{l}\right) \exp \left\{-\frac{1}{2} \sum_{k=m}^{l}(2 i(k)+2)^{2 p} z_{k}^{2}\right\} d \bar{z} \\
& =\frac{1}{(2 \pi)^{(l-m) / 2)}} \int_{\mathbb{R}^{l-m}} C_{\varepsilon_{m}, \ldots, \varepsilon_{l}}\left(\frac{z_{m}}{(2 i(m)+2)^{p}}, \ldots, \frac{z_{l}}{(2 i(l)+2)^{p}}\right) \exp \left\{-\frac{1}{2} \sum_{k=m}^{l} z_{k}^{2}\right\} d \bar{z} .
\end{aligned}
$$

Therefore

$$
1-\int_{\Omega} \exp \left\{-\frac{1}{2} \sum_{k=m}^{l}(2 i(k)+2)^{-2 p} X_{k}^{2}\right\} d P
$$




$$
\begin{aligned}
& =\frac{1}{(2 \pi)^{((l-m) / 2)}} \int_{\mathbb{R}^{l-m}}\left(1-C_{\varepsilon_{m}, \ldots, \varepsilon_{l}}\left(\frac{z_{m}}{(2 i(m)+2)^{p}}, \ldots, \frac{z_{l}}{(2 i)(l)+2)^{p}}\right)\right) \exp \left\{-\frac{1}{2} \sum_{k=m}^{l} z_{k}^{2}\right\} d \bar{z} \\
& =\frac{1}{(2 \pi)^{((l-m) / 2)}} \int_{\mathbb{R}^{l-m}}\left(1-\exp \left\{-\frac{1}{2} \sum_{k=m}^{l} \frac{\sigma_{j(k)}^{2} z_{k}^{2}}{(2 i(k)+2)^{2 p}}\right\}\right) \exp \left\{-\frac{1}{2} \sum_{k=m}^{l} z_{k}^{2}\right\} d \bar{z} \\
& \leqslant \frac{1}{(2 \pi)^{((l-m) / 2)}} \int_{\mathbb{R}^{l-m}} \sum_{k=m}^{l} \frac{\sigma_{j(k)}^{2} z_{k}^{2}}{(2 i(k)+2)^{2 p}} \exp \left\{-\frac{1}{2} \sum_{k=m}^{l} z_{k}^{2}\right\} d \bar{z} \\
& =\sum_{k=m}^{l} \frac{\sigma_{j(k)}^{2} z_{k}^{2}}{(2 i(k)+2)^{2 p}}=\sum_{k=m}^{l} \frac{\sigma_{j(k)}^{2} z_{k}^{2}}{(2 i(k)+2)^{2}} .
\end{aligned}
$$

Since

$$
\sum_{k=1}^{\infty} \frac{\sigma_{j(k)}^{2} z_{k}^{2}}{(2 i(k)+2)^{2}}=\sum_{j=1}^{\infty} \sigma_{j}^{2} \sum_{i=1}^{\infty} \frac{1}{(2 i+2)^{2}}<\infty
$$

as a product of absolutely convergent series, we let $l \rightarrow \infty$ and apply the Lebesgue dominated convergence theorem. We have

$$
1-\int_{\Omega} \exp \left\{-\frac{1}{2} \sum_{k=m}^{\infty}(2 i(k)+2)^{-2 p} X_{k}^{2}\right\} d P \leqslant \sum_{k=m}^{\infty} \frac{\sigma_{j(k)}^{2}}{(2 i(k)+2)^{2}} .
$$

Hence the assertion follows.

End of the Proof of Theorem 1. Given $\varepsilon>0$ we use Lemma 1 to choose $m \in \mathbb{N}$ so that for any $p \in \mathbb{N}$

$$
\begin{aligned}
P\left\{\sum_{k=1}^{\infty}(2 i(k)+2)^{-2 p} X_{k}^{2}<\infty\right\}=\int_{\left\{\sum_{k=m}^{\infty}(2 i(k)+2)^{-2 p} X_{k}^{2}<\infty\right\}} 1 d P \\
\geqslant \int_{\left\{\sum_{k=m}^{\infty}(2 i(k)+2)^{-2 p} X_{k}^{2}<\infty\right\}} \exp \left\{-\frac{1}{2} \sum_{k=m}^{\infty}(2 i(k)+2)^{-2 p} X_{k}^{2}\right\} d P \geqslant 1-\varepsilon .
\end{aligned}
$$

Hence

$$
P\left\{\sum_{k=1}^{\infty}(2 i(k)+2)^{-2 p} X_{k}^{2}<\infty\right\}=1 \text {. }
$$

Define

$$
X(\omega)=\sum_{k=m}^{\infty} X_{k}(\omega) \varepsilon_{k}, \quad \omega \in \Omega .
$$

The mapping $X: \Omega \rightarrow S^{\prime} \otimes H$ is measurable. Let $\mathfrak{m}_{Q}=P \circ X^{-1}$. It is a probability Borel measure on $S^{\prime} \otimes H$.

By (3) we have

$$
C_{Q}\left(P_{\varepsilon_{1}, \ldots, \varepsilon_{n}} \eta\right)=\int_{\Omega} \exp \left\{i\left[P_{\varepsilon_{1}, \ldots, \varepsilon_{n}} X, \eta\right]\right\} d P
$$


Since $P_{\varepsilon_{1}, \ldots, \varepsilon_{n}} \eta \rightarrow \eta$ as $n \rightarrow \infty$ in $S \otimes H$ and $C_{Q}$ is continuous, by Lebesgue's dominated convergence theorem we have

$$
\int_{\Omega} \exp \left\{i\left[P_{\varepsilon_{1}, \ldots, \varepsilon_{n}} X, \eta\right]\right\} d P \longrightarrow \int_{\Omega} \exp \{i[X, \eta]\} d P, \quad n \rightarrow \infty .
$$

Hence we obtain

$$
C_{Q}(\eta)=\int_{\Omega} \exp \{i[X, \eta]\} d P=\int_{S^{\prime} \otimes H} \exp \{i[\omega, \eta]\} d \mathfrak{m}(\omega) .
$$

REMARK. Note that $\mathfrak{m}_{Q}\left(S_{-p} \otimes H\right)=1$ for any $p \geqslant 1$. Hence, $\mathfrak{m}_{Q}$ is supported by $S_{-1} \otimes H$.

\section{4. $Q$-White NOISE MEASURE ON $S^{\prime}(H)$}

Consider the space $S^{\prime}(H)$ of $H$-valued distributions. It consists of all linear continuous operators from $S$ to $H$. We write $\omega(\xi)$ for $\omega \in S^{\prime}(H)$ evaluated against $\xi \in S$. For any $\omega=\sum_{j=1}^{\infty} \omega_{j} \otimes e_{j} \in S^{\prime} \otimes H$ we define $J \omega \in S^{\prime}(H)$ by

$$
J \omega(\xi)=\sum_{j=1}^{\infty}\left\langle\omega_{j}, \xi\right\rangle e_{j}, \quad \xi \in S .
$$

Since the mapping $J: S^{\prime} \otimes H \rightarrow S^{\prime}(H)$ is an isomorphism, we identify $\omega \in S^{\prime} \otimes H$ with $J \omega \in S^{\prime}(H)$ and use the same notation. So we write

$$
\omega(\xi)=\left(\sum_{j=1}^{\infty} \omega \otimes e_{j}\right)(\xi)=\sum_{j=1}^{\infty}\langle\omega, \xi\rangle e_{j} .
$$

Denote by $\mathcal{B}$ the $\sigma$-field in $S^{\prime}(H)$ defined by $\mathcal{B}=J(\mathfrak{B})$. Obviously $\mathcal{B}$ coincides with the Borel $\sigma$-field in $S^{\prime}(H)$. For any $A \in \mathcal{B}$ let $\mu_{Q}(A)=\mathrm{m}_{Q}(B)$ where $B$ satisfies $A=J(B)$.

Let $\omega \in S^{\prime}(H), \xi \in S, h=\sum_{j=1}^{\infty} h_{j} e_{j} \in H$. Then we have

$$
(\omega(\xi), h)_{H}=\left(\left(\sum_{j=1}^{\infty} \omega \otimes e_{j}\right)(\xi), h\right)_{H}=\sum_{j=1}^{\infty}\left\langle\omega_{j}, \xi\right\rangle h_{j}=\sum_{j=1}^{\infty}\left\langle\omega_{j}, h_{j} \xi\right\rangle=\left[\omega, \xi_{h}\right] .
$$

Here $\xi_{h}=\sum_{j=1}^{\infty} h_{j} \xi \otimes e_{j} \in S \otimes H$ since for any $p \in \mathbb{N}$ we have

$$
\sum_{j=1}^{\infty}\left|h_{j} \xi\right|_{p}^{2}=|\xi|_{p}^{2} \sum_{j=1}^{\infty} h_{j}^{2}<\infty
$$


Hence the following equality holds true

$$
\begin{aligned}
& \int_{S^{\prime}(H)} \exp \left\{i(\omega(\xi), h)_{H}\right\} d \mu_{Q}(\omega)=\int_{S^{\prime} \otimes H} \exp \left\{i\left[\omega, \xi_{h}\right]\right\} d \mathfrak{m}_{Q}(\omega) \\
& =\exp \left\{-\frac{1}{2}\left[(I \otimes Q) \xi_{h}, \xi_{h}\right]_{0}\right\}=\exp \left\{-\frac{1}{2} \sum_{j=1}^{\infty} \sigma_{j}^{2}\left|h_{j} \xi\right|_{0}^{2}\right\} \\
& =\exp \left\{-\frac{1}{2}|\xi|_{0}^{2}(Q h, h)_{H}\right\} .
\end{aligned}
$$

Consider the probability space $\left(S^{\prime}(H), \mathcal{B}, \mu_{Q}\right)$. Define a generalised $H$-valued stochastic process $\{\mathbb{W}(\xi, \omega), \xi \in S\}$ by

$$
\mathbb{W}(\xi, \omega)=\omega(\xi) .
$$

It follows from the equality (5) that for any $h \in H$ the $\mathbb{R}$-valued generalised stochastic process $\left\{(\mathbb{W}(\xi, \omega), h)_{H}, \xi \in S\right\}$, which can be regarded as a projection of $\mathbb{W}$ onto $\operatorname{Sp}\{h\}$, is a smoothed white noise with variance $(Q h, h)_{H}$. On the other hand, for any $\xi \in S$, $\mathbb{W}(\xi, \cdot)$ is an $H$-valued Gaussian random variable with mean 0 and covariance operator $|\xi|_{0}^{2} Q$. Therefore we refer to $\left(S^{\prime}(H), \mathcal{B}, \mu_{Q}\right)$ as the $H$-valued $Q$-white noise space. The generalised stochastic process $\mathbb{W}(\xi, \omega)$ is referred to as the $H$-valued $Q$-white noise.

Consider the space $L^{2}\left(S^{\prime}(H) ; H\right)$ of square (Bochner) integrable $H$-valued random variables defined on $S^{\prime}(H)$. For any $\xi \in S$ random variable $\mathbb{W}(\xi, \cdot): S^{\prime}(H) \rightarrow H$ belongs to $L^{2}\left(S^{\prime}(H) ; H\right)$. We have

$$
\|\mathbb{W}(\xi, \cdot)\|_{L^{2}\left(S^{\prime}(H) ; H\right)}^{2}=\operatorname{Tr} Q \cdot\|\xi\|_{S_{0}}^{2} .
$$

Define stochastic process $\{W(t) \mid t \geqslant 0\}$ on $\left(S^{\prime}(H), \mathcal{B}, \mu_{Q}\right)$ by

$$
W(t)(\omega)=\omega\left(\chi_{[0 ; t]}\right):=\lim _{n \rightarrow \infty} \omega\left(\theta_{n}\right),
$$

where limit is taken in $L^{2}\left(S^{\prime}(H) ; H\right)$ and $\left\{\theta_{n}\right\}_{n=1}^{\infty} \subset S$. is a sequence convergent to $\chi_{[0 ; t]}$ in $L^{2}(\mathbb{R})$. Existence of the limit in (7) and its independence of the choice of $\left\{\theta_{n}\right\}_{n=1}^{\infty} \subset S$ follow from (6). It is not difficult to check that $W(t)$ is a $Q$-Wiener process. Its trajectories are continuous $H$-valued functions.

For any $\xi \in S$ we have

$$
\begin{aligned}
-\int_{\mathbb{R}} W(t) \xi^{\prime}(t) d t & =-\int_{\mathbb{R}} \omega\left(\chi_{[0 ; t]}\right) \xi^{\prime}(t) d t=\omega\left(-\int_{\mathbb{R}} \chi_{[0 ; t]}(s) \xi^{\prime}(t) d t\right) \\
& =\omega\left(-\int_{s}^{\infty} \xi^{\prime}(t) d t\right)=\omega(\xi) .
\end{aligned}
$$

Thus, $\mathbb{W}$ can be regarded as a generalised derivative of $W(t)$ (in $S^{\prime}(H)$ sense). 
Let $W_{0}(t)$ be defined by

$$
W_{0}(t)= \begin{cases}W(t), & t \geqslant 0 \\ 0, & t<0\end{cases}
$$

Its trajectories are continuous with probability 1 . Define generalised stochastic process $\mathbb{W}_{0}$ by $\mathbb{W}_{0}(\xi, \omega)=W_{0}^{\prime}(\xi)$, where derivative is understood in the generalised sense:

$$
\mathbb{W}_{0}(\xi, \omega)=-\int_{\mathbb{R}} W_{0}(t) \xi^{\prime}(t) d t=-\int_{0}^{\infty} W(t) \xi^{\prime}(t) d t .
$$

It is natural to call $\mathbb{W}_{0}$ the $Q$-white noise with support in $[0, \infty)$, or the $Q$-white noise starting at $t=0$.

\section{Equations WITH ADDITIVE NOISE}

Let $X, Y$ and $H$ be separable Hilbert spaces. Consider the equation

$$
P * U=F+B W_{0},
$$

where $P \in D_{+}^{\prime}(\mathcal{L}(X ; Y)), U \in D_{+}^{\prime}(X), F \in D_{+}^{\prime}(Y), B \in \mathcal{L}(H ; Y)$ and $\mathbb{W}_{0}$ is the $H$ valued $Q$-white noise with support in $[0 ; \infty)$, on the probability space $\left(S^{\prime}(H), \mathcal{B}, \mu_{Q}\right)$. Let $P$ have a convolution inverse $G \in D_{+}^{\prime}(\mathcal{L}(Y ; X))$. Then the generalised stochastic process $\{U(\xi, \omega), \xi \in S\}$, defined by

$$
U(\xi, \omega):=(G * F)(\xi)+\left(G * B \mathbb{W}_{0}\right)(\xi, \omega)
$$

is the unique solution of (8). Convolution $G * B \mathbb{W}_{0}$ is well defined since $B \mathbb{W}_{0}(\cdot, \omega)$ has support bounded from below for any $\omega \in S^{\prime}(H)$ (see [1]).

Now we consider a particular example of $P$. Let $A$ be a closed linear operator acting in $Y$ and $X=[D(A)]$ be the domain of $A$, endowed with the graph-norm. Then

$$
P=\delta^{\prime} \otimes I-\delta \otimes A \in D_{+}^{\prime}(\mathcal{L}(X ; Y))
$$

Define $F \in D_{+}^{\prime}(Y)$ by

$$
F(\xi):=\xi(0) u^{0}+\int_{0}^{\infty} \xi(t) f(t) d t, \quad \xi \in D, f \in L_{1}^{\mathrm{loc}}(\mathbb{R}, Y), u^{0} \in Y
$$

Then the Cauchy problem

$$
u^{\prime}(t)=A u(t)+f(t), \quad t>0, \quad u(0)=u^{0}
$$

can be written in the form

$$
P * U=F
$$


(see $[\mathbf{1}, 4]$ ). If the right-hand side of (11) is perturbed by a white noise term, then it is natural to write it in form (8) in the space of distributions $S^{\prime}(H)$.

Let $A$ in (10) be the generator of a $C_{0}$-semigroup $\{S(t), t \geqslant 0\}$. Then the convolution inverse to $P$ is

$$
G(\xi)=\int_{0}^{\infty} \xi(t) S(t) d t
$$

and formula (9) becomes

$$
\begin{aligned}
U(\xi, \omega)=\int_{0}^{\infty} \xi(t) S(t) u^{0} d t+\int_{0}^{\infty} \int_{0}^{t} S(t-s) f(s) d s & \xi(t) d t \\
& -\int_{0}^{\infty} \int_{0}^{t} S(t-s) B \omega\left(\chi_{[0 ; s]}\right) d s \xi(t) d t
\end{aligned}
$$

If $A$ is the generator of an exponentially bounded $n$-times integrated semigroup $\{V(t), t \geqslant 0\}$, then the convolution inverse to $P$ has the form

$$
G(\xi)=(-1)^{n} \int_{0}^{\infty} \xi^{(n)}(t) V(t) d t
$$

and formula (9) becomes

$$
\begin{aligned}
U(\xi, \omega)=(-1)^{n} \int_{0}^{\infty} \xi^{(n)}(t) V(t) u^{0} d t & +(-1)^{n} \int_{0}^{\infty} \int_{0}^{t} V(t-s) f(s) d s \xi^{(n)}(t) d t \\
& +(-1)^{n+1} \int_{0}^{\infty} \int_{0}^{t} V(t-s) B \omega\left(\chi_{[0 ; s]}\right) d s \xi^{(n+1)}(t) d t
\end{aligned}
$$

\section{REFERENCES}

[1] H.O. Fattorini, The Cauchy Problem (Cambridge University Press, Cambridge, 1983).

[2] T. Hida, Brownian motion, Applications of Mathematics 11 (Springer-Verlag, Berlin, Heidelberg, New York, 1980).

[3] H. Kuo, Gaussian Measures in Banach Spaces, Springer Lecture Notes in Maths. 643 (Springer-Verlag, Berlin, Heidelberg, New York, 1975).

[4] I. Melnikova and A. Filinkov, Abstract Cauchy problems: Three approaches (Chapman \& Hall, Boca Raton, 2001).

Ural State Technical University

Yekaterinburg

Russia

e-mail: mxa@mail.ur.ru 